\title{
Techniques for the design of human error tolerant systems
}

\author{
Full day tutorial \\ P. Wright, B. Fields, A. Dearden, M. Harrison \\ Presented by: P. Wright \& B. Fields \\ Human-Computer Interaction Group \\ Department of Computer Science \\ University of York \\ York YO1 5DD, UK. \\ Phone: +44 $1904432741 \quad$ Fax: +44 1904432767 \\ Email: \{pcw,bob\}@minster.york.ac.uk
}

KEYWORDS: designing for error, human-error tolerance, human-reliability analysis, safety

\section{INTRODUCTION}

Interactive systems need to be robust to human error and provide for easy recovery. This tutorial describes techniques that can help designers to build human-error tolerance into their systems.

Have you ever set your VCR and returned only to find it missed your favourite program? Have you ever been frustrated when trying to set the time on a digital watch? Do any of the systems your company designs lead to similar frustrations for their users? What can you do to avoid these problems?

In safety-critical systems, such as power stations, chemical plants, or aircraft cockpits, the consequences of human error can be catastrophic rather than just frustrating. Methods for analysing designs to discover the potential for human error are critical in these fields.

\section{TUTORIAL CONTENT}

This tutorial will equip you with some simple techniques for tackling human error issues in design, provide a framework for thinking about error, and give pointers to where more information can be found. The areas covered are listed below.

\section{Analysing the work context}

One starting point for error analysis is to understand the tasks that the user intends to perform and the situation in which work is carried out. The tutorial discusses how tasks can be studied and represented, with a focus on one simple task description technique, Hierarchical Task Analysis. The importance of the situation or scenario, and ways of describing scenarios, will also be discussed.

Participants will be given an opportunity to try out these ideas by applying them to simple, everyday systems.

\section{Analysing the system context}

Understanding the work being carried out is an essential part of identifying potential error problems. The interactive systems and technology being used is another important contextual factor. This section of the tutorial introduces a collection of concepts and techniques that can help the analyst in isolating aspects of an interface design that could lead to errors (such as moding problems or display complexity). 


\section{Identifying error and breakdown Cognitive analysis of error}

The tutorial will consider some of the accounts of error that are available from cognitive psychology, and how these can be used to identify and explain potential errors, using the contextual factors discussed earlier.

\section{Behavioural analysis of error}

A cognitive view of error causation may help to identify where problems might occur and how to design for them, but does not give the analyst a basis for deciding how serious errors will be. For this we need to look to a more detailed account of how errors are manifested in behaviour.

Given a description of a task, it is possible to generate some error scenarios by systematically applying a checklist of guide words to user actions.

\section{Identifying significant errors}

Not all errors are serious, and designers need to prioritise their work to ensure that the errors that have the greatest impact are the ones that are dealt with first. This part of the tutorial will discuss approaches that can be used to determine the most important errors. The emphasis will be on systematic ways of exploring the consequences of an error, and of determining the relative significance of different errors.

Participants will be given an opportunity to apply the knowledge of cognitive pre-cursors of error, and methods for exploring error consequences with a simple case study.

\section{Designing to avoid error}

The aim of error analysis is to develop designs that are resistant and tolerant to error. There are no automatic rules that will generate good designs, but this part of the tutorial discusses some ways in which a design can be made more or less tolerant of error. The tutorial covers 'localised' techniques such as confirmation dialogues and recovery mechanisms; ways of thinking about how information is presented to aid error detection and recovery; as well as approaches to conceptual design, i.e. the earliest stages of design.

\section{The expanding context}

The final part of the tutorial explores some of the most difficult problems in understanding error. Some events that lead to undesirable consequences are difficult to account for using only the tools of behavioural analysis and cognitive analysis. These events involve social, historical and cultural issues. The tutorial highlights some of these issues using examples of accidents and incidents in safety critical systems.

\section{INTENDED AUDIENCE}

The tutorial is intended for system designers and engineers involved in the design of user interfaces as well as human-computer interaction researchers. It is intended that the content of the tutorial be relevant not only to practitioners in the world of safety critical systems, but also to developers and systems where human error may cause problems. Examples used include everyday domestic items and high-volume products where usability is an important issue.

No prior experience will be assumed of task analysis, human reliability analysis or the psychology of human error.

\section{ABOUT THE AUTHORS}

Peter Wright, Bob Fields, Michael Harrison and Andy Dearden bring substantial academic and industrial experience of Human Computer Interaction and Human Reliability Analysis. Wright, Fields and Harrison, both software engineers, are members of the British Aerospace (BAe) Dependable Computing System Centre at the University of York. The centre was set up by BAe to carry out long-term research and focussing on safety and dependability in aerospace systems. Dearden is a member of the human-computer interaction group at York, and is investigating concepts of risk, impact and utility in human-machine interface design.

Between them they have conducted studies of human-error issues in several different domains, ranging from aircraft flight decks, to government agencies, and emergency services' control rooms.

Their research work is focussed on developing techniques to aid designers in building error tolerant and error resistant systems. 